\title{
Evaluation of the efficacy and safety of two types of rhBMP-2- soaked bone graft materials in extraction socket preservation
}

\author{
Kim Sang Yun ${ }^{1 *}$, Kim Young-Kyun ${ }^{1,2}$, Jo Deuk-Won ${ }^{3}$ \\ 1 Department of Oral and Maxillofacial Surgery, Section of Dentistry, Seoul National University Bundang Hospital, \\ Seongnam , Korea \\ ${ }^{2}$ Department of Dentistry \& Dental Research Institute, School of Dentistry, Seoul National University, Seoul, Korea \\ ${ }^{3}$ Department of prosthodontics, Section of Dentistry, Seoul National University Bundang Hospital, Seongnam , Korea
}

\section{Introduction}

Recombinant human bone morphogenetic protein-2 (rhBMP2 ), which is a potent osteoinductor, has long been considered a promising avenue for bone regeneration, and has been commercially available for the treatment of oral and maxillofacial hard-tissue defects.

\section{Purpose}

The aim of this study was to evaluate the safety and effect of two types of rhBMP-2-soaked bone graft materials on extraction socket preservation.

\section{Materials and Methods}

Between October 2015 and September 2016, bone graft was performed in 64 patients scheduled to receive implantation after single tooth extraction.

2 types of rhBMP-2-soaked bone graft materials

1) O-BMP (Osstem Implant Co., Busan, Korea; scaffold: collagen sponge)

: experimental group $(\mathrm{n}=32)$

2) Cowell BMP (Cowell medi, Busan, Korea; scaffold: $\beta$ TCP)

: control group $(n=32)$

After covering the resorbable collagen membrane, the flap was closed.

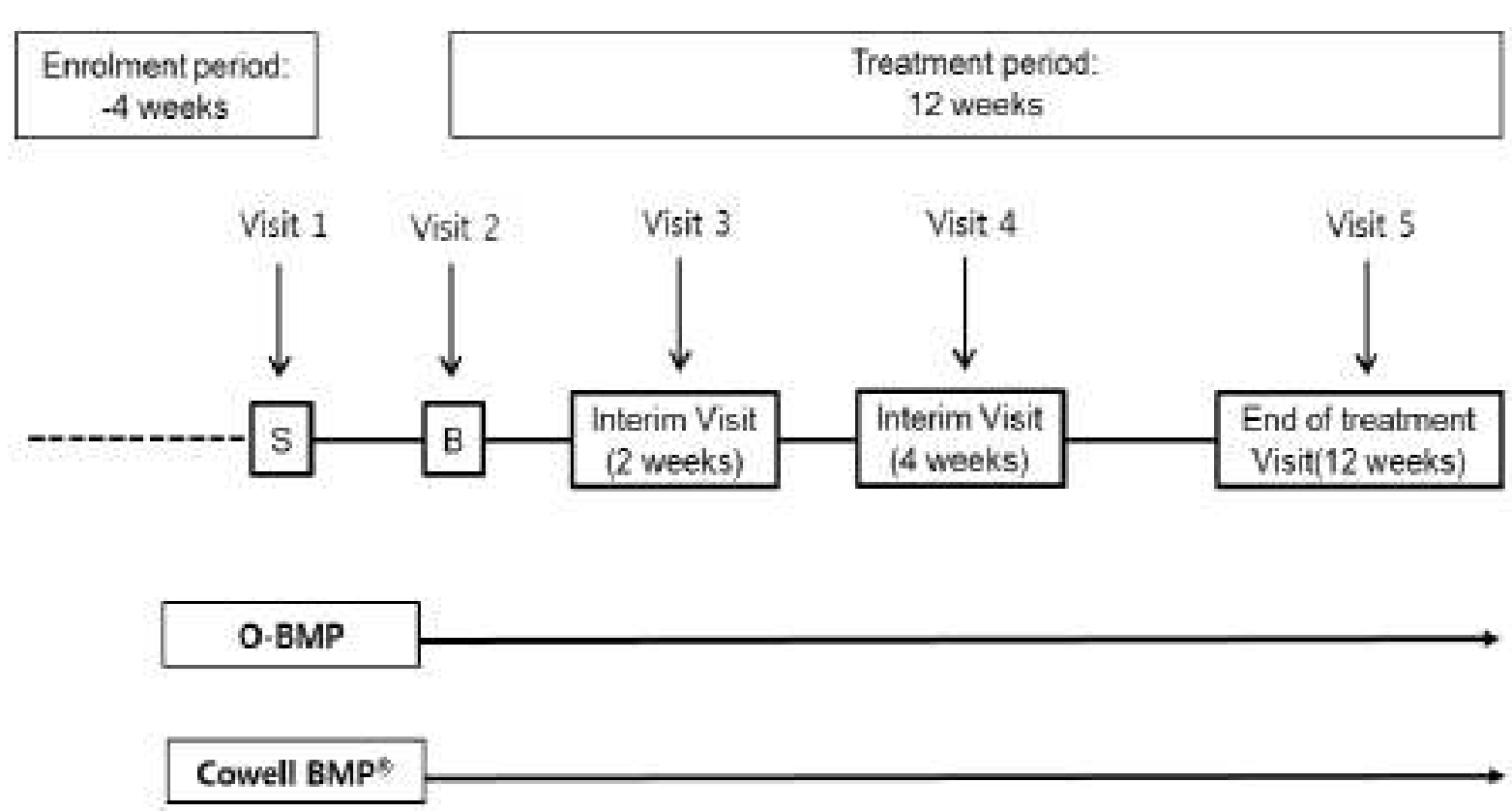

Before and 12 weeks after the operation, panoramic radiograph and cone-beam computed tomography (CBCT) was used to assess the changes in bone height, and the widths at $25 \%, 50 \%$, and $75 \%$ of the extraction socket levels.

\section{Histological evaluation :}

Patients who agreed to undergo biopsy were evaluated for histological healing by obtaining tissue specimens with a trephine bur at the time of implant placement. (3-4 months after bone grafting).

\section{Results}

\section{Clnical results:}

Both the experimental group and the control group showed good healing without specific complications such as infection.

\section{Radiographic results:}

CBCT evaluation of the width and height change of the extraction socket revealed that the amount of bone resorption in the experimental and control groups was not large and that the shape of the extraction socket was well maintained.

\section{Histological results:}

Both groups showed good healing state without inflammatory reaction and new bone formation increased with time.

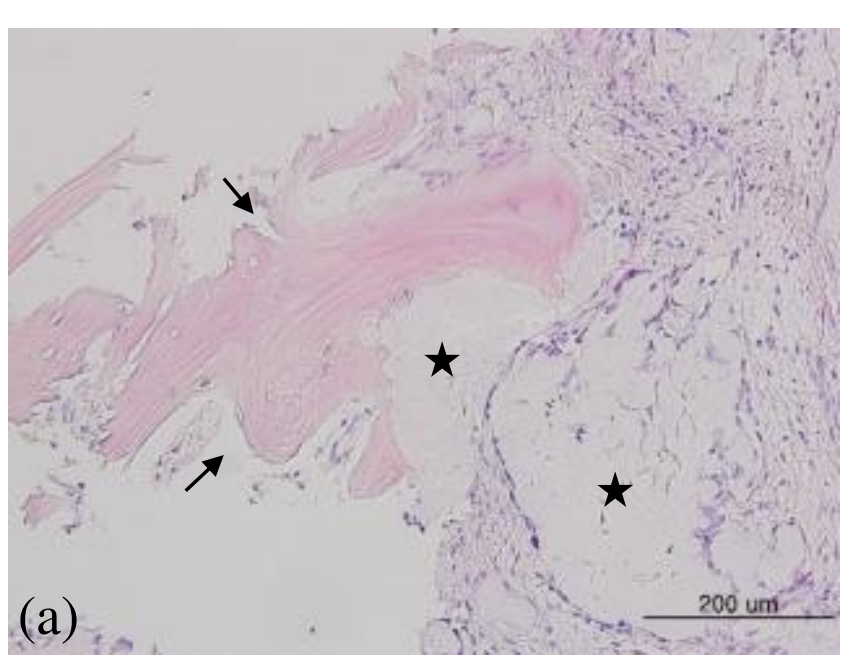

(a) COWELL BMP

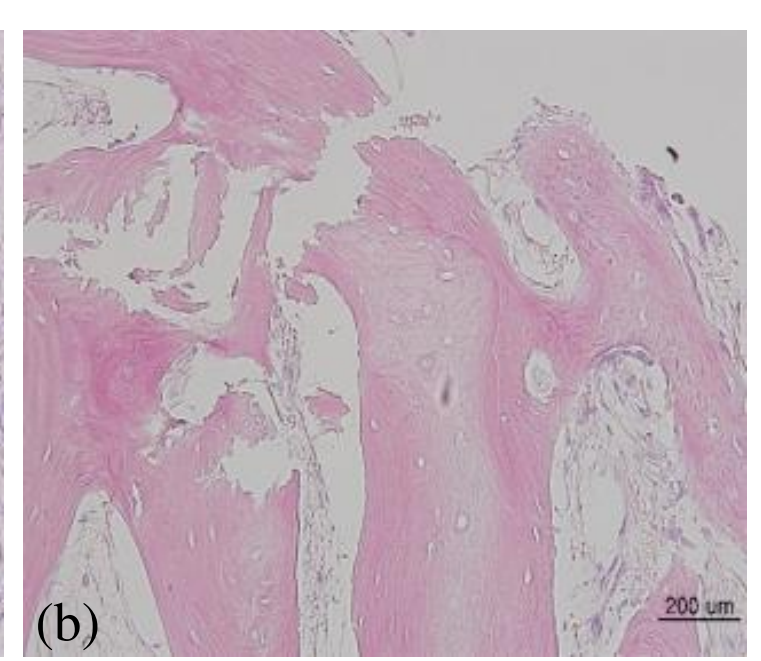

(b) O-BMP

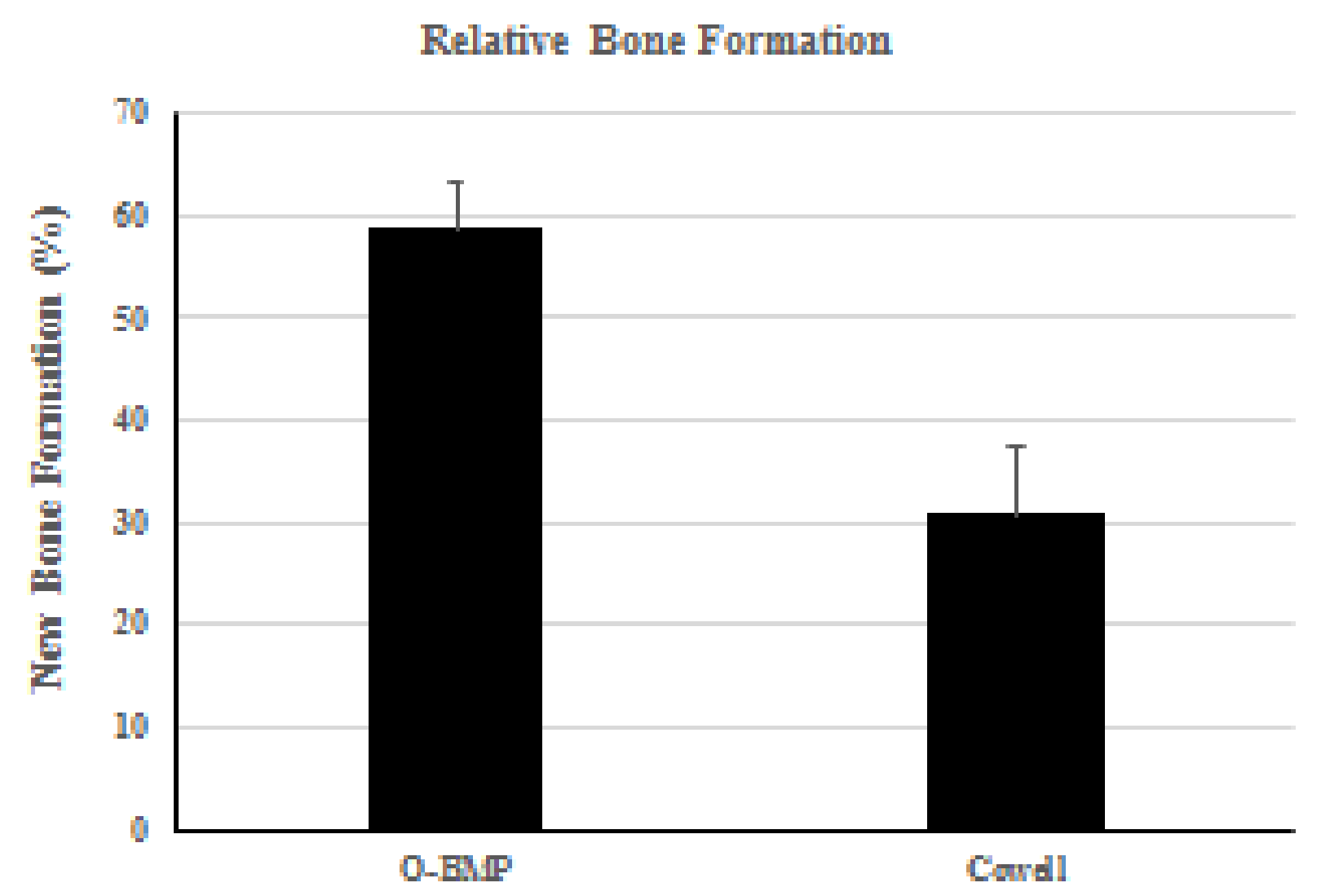

\section{Conclusions}

Compared with Cowell BMP®, which has been proven effective and safe, O-BMP used in extraction socket preservation resulted in clinically and histologically better healing and preservation. 